\title{
Normal Range of Thoracic Kyphosis in Male School Children
}

\author{
MohammadBagher Shamsi, ${ }^{1,2}$ Korosh Veisi, ${ }^{3}$ Loghman Karimi, ${ }^{4}$ \\ Javad Sarrafzadeh, ${ }^{2}$ and Farid Najafi ${ }^{5}$ \\ ${ }^{1}$ Rehabilitation and Sport Medicine Department, School of Paramedicine, Kermanshah University of Medical Sciences, \\ Kermanshah 6715847141, Iran \\ ${ }^{2}$ Physiotherapy Department, School of Rehabilitation Sciences, Iran University of Medical Sciences, Mohseni Square, \\ Tehran 439115875, Iran \\ ${ }^{3}$ Department of Sport Management, Kurdestan Research and Science Branch, Islamic Azad University, Sanandaj 6616964353, Iran \\ ${ }^{4}$ Saqqez Education Office, Saqqez 6681763761, Iran \\ ${ }^{5}$ Kermanshah Health Research Center, School of Population Health, Kermanshah University of Medical Sciences, \\ Kermanshah 6715847141, Iran
}

Correspondence should be addressed to MohammadBagher Shamsi; mshamsi@kums.ac.ir

Received 29 January 2014; Accepted 12 March 2014; Published 6 April 2014

Academic Editors: J. Dubousset and G. Papachristou

Copyright (C) 2014 MohammadBagher Shamsi et al. This is an open access article distributed under the Creative Commons Attribution License, which permits unrestricted use, distribution, and reproduction in any medium, provided the original work is properly cited.

\begin{abstract}
Background. Although there are frequent studies about normal range of thoracic kyphosis, there is still a controversy about the exact values of this curve. In nine reported studies on 10 to 20 years of age boys, the value ranged from $25.1^{\circ}$ to $53.3^{\circ}$. Objective for the Study. The aim of the present research was investigation of normal ranges of thoracic kyphosis in school children in Kermanshah, western Iran. Methods. 582 male students aged 13 to 18 years old using cluster random sampling were recruited from schools in Kermanshah city, 97 students for each age. Thoracic curves were measured using the flexicurve method. Results. Mean thoracic kyphosis for whole population was $35.49^{\circ} \mathrm{SD} 7.83$ and plus or minus two standard deviations ranged from $19.83^{\circ}$ to $51.15^{\circ}$. It increased gradually from 13 to 16 and then there was a little decrease to 18 years. Mean values for each age (13-16) were $13\left(34.41 \mathrm{SD} 7.47^{\circ}\right), 14\left(34.86 \mathrm{SD} 8.29^{\circ}\right)$, $15\left(35.79 \mathrm{SD} 7.93^{\circ}\right), 16\left(36.49 \mathrm{SD} 7.85^{\circ}\right), 17\left(35.84 \mathrm{SD} 8.33^{\circ}\right)$, and $18\left(35.55 \mathrm{SD} 7.07^{\circ}\right)$. Conclusions. Our results are in agreement with previous reports and can be used as normal values for local and regional purposes.
\end{abstract}

\section{Introduction}

The thoracic angle is the primary curve of the vertebral column which is comprised of 12 vertebrae [1]. The thoracic kyphosis angle increases with age and the increase is greater in females than in males $[2,3]$. Hyperkyphosis or increase in thoracic curve greater than normal range is one of prevalent spinal disorders. Biomechanical data suggest that an increase in the thoracic kyphosis may be associated with significantly higher spinal loads and trunk muscle force in upright stance and this might accelerate degenerative process which in turn leads to further spinal dysfunction and pain [4]. An increase in thoracic kyphosis has also been associated with diminished physical function [5], impairment of respiratory function $[6,7]$, an increase in cervical pain $[8,9]$, headaches $[10]$, and shoulder discomforts such as subacromial pain syndrome $[11,12]$.

In spite of frequent studies on normal range of thoracic angle, there is a controversy about the magnitude of this curve. For example in Willner and Johnson [13] study the least pronounced kyphosis was seen at the age of 10-12 years and mean kyphosis angle in 8 and 16 years was $35^{\circ}$ and $44^{\circ}$, respectively [13]. Propst Proctor and Bleck [14] in a study on children aged from 2 to 20 years old reported an angle of $27^{\circ}$ (range of 21-33 ${ }^{\circ}$ ) as normal. The magnitude of thoracic kyphosis in the study of Voutsinas and MacEwen [15] averaged $38.1^{\circ}$ in white boys and $34.3^{\circ}$ in black boys and $38.5^{\circ}$ in white girls and $36.9^{\circ}$ in black girls. In adults, kyphosis curve values varied according to different investigators, with the range of $\sim 35^{\circ}-37^{\circ}[16,17]$, but such studies were conducted 


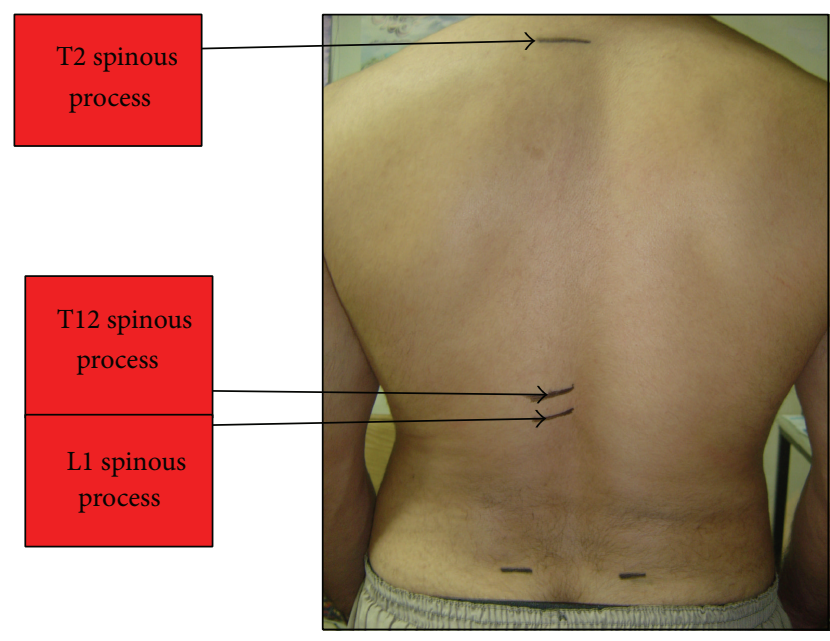

FIgURE 1: Locating T2, T12, and L1 spinous processes.

in heterogeneous populations. Boseker et al. [18] using \pm two standard deviations reported that normal kyphosis is ranged from $20^{\circ}$ to $50^{\circ}$.

Such wide variations may be partly related to factors that affect spinal curve measurement such as difference in the instrument used and sample characteristic (age, sex, ...), and limitations researchers have been faced with and partly related to factors that have been claimed to have influence on the curve such as age [19], sex [19], geographical region [17], and race [15].

Although there are quite different studies around the world on ranges of kyphosis, it seems that each region needs its specific values. The aim of this study was to define normal kyphosis range in 13-18-year-old male students living in Kermanshah, a city in the west of Iran.

\section{Materials and Methods}

The Ethical Board of Kermanshah University of Medical Sciences approved this study. For this purpose, 582 subjects were chosen across male students aged 13 to 18 years living in Kermanshah. Using random cluster sampling 6 guidance schools, 6 high schools were chosen. In each school, for every age group (13-18), at least 16 students were defined randomly. Exclusion criteria were having neuromuscular disorders, scoliosis, history of surgery, or any diseases on spinal column. In addition, if the students suffered from severe kyphosis, they were excluded.

All participants signed an informed consent form. In order to measure the kyphosis, they were asked to take off dresses and shoes. For all students weight and height were measured. Using Adams test, participants were asked about history and sign of spinal surgery. For measuring the kyphosis, students were asked to stand comfortable and look forward in a way that body weight would be divided equally on both feet (arms beside the body). In this position, using surface anatomy and by palpation, spinous processes of 2 nd and 12th thoracic vertebrae (T2 and T12) were determined and marked by a marker (Figure 1).

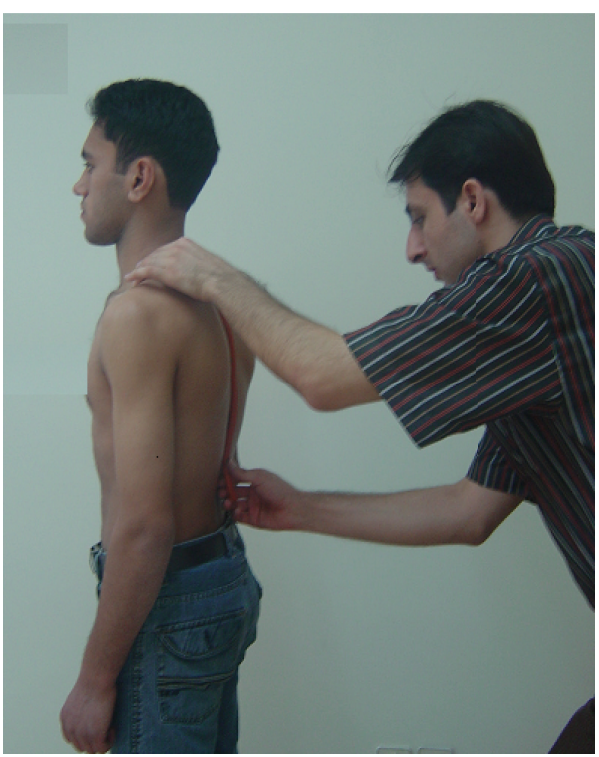

FIGURE 2: Placing flexible ruler on subjects backs for measuring its angle.

A flexible ruler was placed on thoracic curve covering the two marked points and pushed gently on back so there was no gap between the skin and the ruler (Figure 2). Keeping this position, a second coworker marked the adjacent points to T2 and T12 spinous processes on the ruler. Without making any change in the curve induced in the flexible ruler, it was put on a piece of paper. Using a pen, the curve was drawn and those two points were reflected on the paper. After removing the ruler a straight line was drawn connecting T2 and T12 points. The length of this line was measured and called "l". Then distance between the deepest point of the curve and line "l" was measured as " $h$ ". Using the equation below, magnitude of the angle of thoracic curve was measured [20]:

$$
\theta=4 \operatorname{Arctang}\left(\frac{2 h}{l}\right)
$$

Before starting the study, the reliability of the measurements was analysed using intraclass correlation coefficients (ICC). Forty-three subjects were tested 2 times with 5 minute interval. Data were analysed using SPSS version 11.5 software.

\section{Results}

Number of subjects in each age group and their height and weight characteristics are shown in Table 1. Using ANOVA statistic test $(P<0.001)$ it was found that mean height and weight of subjects in age groups were significantly different. By performing the measurement 2 times with 5 minute interval, intraclass correlation coefficients (ICC) were found high (86\%); therefore a good reliability of this test was confirmed. Table 2 shows mean kyphosis angle with 95\% confidence interval in each age group (13-18). Mean thoracic kyphosis for each age group was $13\left(34.41^{\circ}\right), 14\left(34.86^{\circ}\right), 15$ $\left(35.79^{\circ}\right), 16\left(36.49^{\circ}\right), 17\left(35.84^{\circ}\right)$, and $18\left(35.55^{\circ}\right)$. 
TABLE 1: Subject's characteristics (Mean \pm SD).

\begin{tabular}{lccc}
\hline Age groups $(\mathrm{y})$ & Number $(n)$ & Height $(\mathrm{cm})$ & Weight $(\mathrm{kg})$ \\
\hline 13 & 97 & $156.99 \pm 8.76$ & $48.02 \pm 9.97$ \\
14 & 97 & $165.06 \pm 7.42$ & $53.24 \pm 11.06$ \\
15 & 97 & $168.07 \pm 7.44$ & $56.32 \pm 9.82$ \\
16 & 97 & $174.28 \pm 6.22$ & $64.68 \pm 10.23$ \\
17 & 97 & $175.70 \pm 5.88$ & $67.14 \pm 7.62$ \\
18 & 97 & $176.32 \pm 6.19$ & $69.46 \pm 8.09$ \\
\hline Sum & 582 & & \\
\hline
\end{tabular}

TABLE 2: Mean and normal range of thoracic kyphosis in each age group.

\begin{tabular}{lcc}
\hline Age group (year) & Normal range $^{*}$ & $($ Mean \pm SD) \\
\hline 13 & $19.47 \pm 49.34$ & $34.41 \pm 7.47$ \\
14 & $18.29 \pm 51.44$ & $34.86 \pm 8.29$ \\
15 & $19.92 \pm 51.65$ & $35.79 \pm 7.93$ \\
16 & $20.78 \pm 52.20$ & $36.49 \pm 7.85$ \\
17 & $19.18 \pm 52.50$ & $35.84 \pm 8.33$ \\
18 & $21.42 \pm 49.69$ & $35.55 \pm 7.07$ \\
\hline
\end{tabular}

${ }^{*}$ Normal range in each population is mean \pm 2 SD.

When the whole population is considered, the average kyphosis was $35.49^{\circ} \mathrm{SD} 7.83$, ranging from $12.05^{\circ}$ to $68.84^{\circ}$. This study would indicate that $95 \%$ confidence interval of normal kyphosis was between $19.83^{\circ}$ to $51.15^{\circ}$. Using ANOVA statistic test $(P<0.001)$ it was found that mean kyphosis of subjects in 13-18-year groups was significantly different too.

With increase in age from 13 to 16 years old, the magnitude of mean of kyphosis increased from $34.41^{\circ}$ to $36.49^{\circ}$ and then there was a mild decrease reaching to $35.5^{\circ}$ by the age of 18 years old (Figure 3 ). The least thoracic angle was $12.05^{\circ}$ observed in students who were 13 years old and the biggest was $68.84^{\circ}$ in those who were 16 years old.

\section{Discussion}

The goal of this study was determination of thoracic kyphosis "normalcy" in male children and adolescents ranging from 13 to 18 years of age for regional population.

Most researchers believe that there is an increase in thoracic kyphosis in the age of 10 to 20 in both sexes. Mac-Thiong et al. [21] reported the tendency of kyphosis to increase linearly with age for children and adolescents. Our research confirms this increase from 13 to 18 too. Fon et al. [2] stated that the increase in the kyphotic curve with age is not unexpected, because of the associated changes in the soft tissues and mineral content of the bones with the progression of years. An association of progressive increase in spinal curvatures with gradual compression wedging of the vertebrae and its narrowing of the intervertebral discs has been described. Age-related increases in thoracic kyphosis can be attributed to overloading spinal soft tissue, particularly to the intervertebral disk with aging, during the growth period.

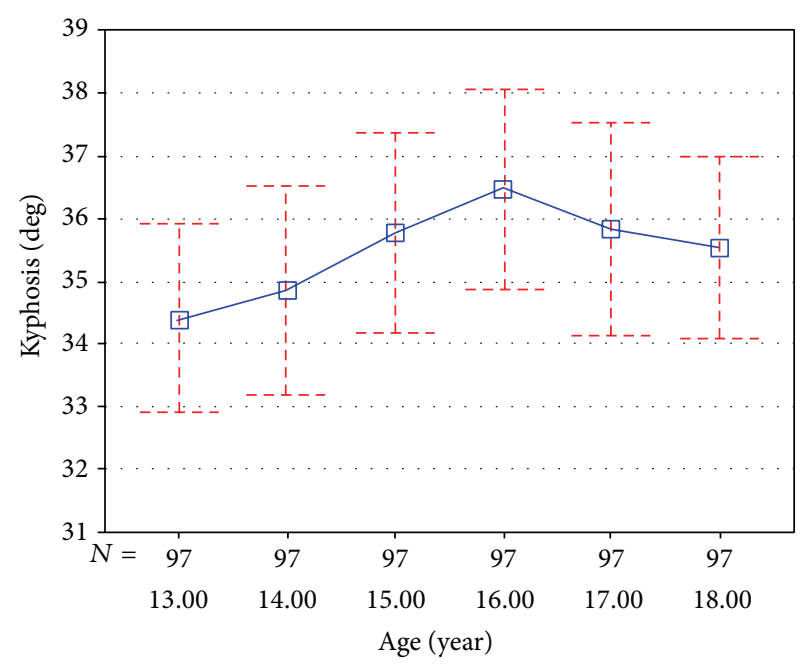

FIGURE 3: Thoracic kyphosis change in age groups $(n=582)$.

Fon et al. [2], Willner and Johnson [13], Voutsinas and MacEwen [15], Boseker et al. [18], Mac-Thiong et al. [21], Poussa et al. [22], and Cil et al. [23] in their articles have reported the least pronounced kyphosis at the age of 10-12 years, but, because we did not choose subjects in this range, the least kyphosis in our study was seen in the 13-year-old group.

The biggest magnitude of mean curve was seen in 16-yearold age group that may be due to pubertal growth. A positive correlation was seen between the velocity of growth and the range of the kyphosis in Willner and Johnson [13], Poussa et al. [22], and Cil et al. [23] studies. In the present study we cannot conclude this correlation because the puberties of subjects were not assessed.

Most researches have shown an age-related trend toward increases in thoracic kyphosis. However, some decreases were observed in isolated groups. For example, Willner and Johnson [13], Poussa et al. [22], and Giglio and Volpon [24] reported some decreases at 15, 14, and 15-16 years of age, respectively. Our subjects demonstrated a decrease in 17-18. The age for this decrease in each research was different which can be due to natural differences in subjects (race, lifestyle, age of puberty related to geographic region, etc.), or margin of errors demonstrated in using different measurement methods. Giglio considered these isolated changes to be a random occurrence.

Radiography (Cobb method) is the most commonly used method to assess sagittal spinal curves. Some studies using radiography including Takemitsu and Harada [25] in a study on 519 students reported $37^{\circ}$ for mean thoracic kyphosis. Fon et al. [2] (measuring superior border of the upper end vertebra as well as along the inferior border of the lower end vertebra) found $25.11^{\circ}$ for 10-19-year-old boys. Probst Proctor and Bleck [14] also measured the T5-12 area in 104 normal children. The mean value was $27^{\circ}$ (range, $2^{\circ}-33^{\circ}$ ). Vedantam et al. [26] measured the thoracic kyphosis from the upper end plate of T3 to the lower end plate of T12 in 88 boys and girls of $10-18$ years of age. Mean angle was $38.00^{\circ}$. Boseker et al. 
[18] in a study on 121 subjects of 5-19 years of age stated the mean angle $33.00^{\circ}$ with normal range (mean \pm 2 SD) of $20-$ $50^{\circ}$. In Mac-Thiong et al. [21] study, values of $38.3^{\circ}$ and $44.2^{\circ}$ were reported for children younger than 10 years old and subjects of 10 years of age or older (both sexes), respectively. And Cil et al. [23] in a study of both male and female showed the average to be $45.8^{\circ}$ for $10-12$ and $53.3^{\circ}$ for $13-15$ years of age.

Although radiography is the most commonly used, this method is not ideal for systematic population studies because of its high cost and exposure of subjects to ionizing radiation. Some radiograph images are difficult to analyze, as it may be hard to precisely identify the beginning of the kyphotic curve because of the shoulder girdle and rib overlap. To overcome these limitations, thoracic curve was clinically evaluated by other instruments.

Willner and Johnson [13] studied the thoracic kyphosis in 1101 healthy children in consecutive age groups between 8 and 16 years of age using spinal pantograph. Their mean values for different ages were $13\left(31.9^{\circ}\right), 14\left(37.1^{\circ}\right), 15\left(35.6^{\circ}\right)$, and $16\left(37.4^{\circ}\right)$. Using the same instrument, Poussa et al. [22] results for boys in $11,12,13$, and 14 years of age were $27.8^{\circ}$, $28.0^{\circ}, 30.9^{\circ}$, and $30.0^{\circ}$, respectively. Widhe [27] measured the thoracic curve using kyphometer on 46 males of 15-16 years and the mean value was $33.7^{\circ}$. And Giglio and Volpon [24] using spinal pantograph showed $33^{\circ}, 38^{\circ}, 35^{\circ}, 32^{\circ}, 36^{\circ}$, and $37^{\circ}$ for thorasic kyphosis in $13,14,15,16,17$, and 18 year old boys, respectively.

To investigate the comparative validity and the intraand interrater reliability of thoracic kyphosis measurements using the flexicurve method, Teixeira and Carvalho [28] conducted a study in which the thoracic kyphosis was evaluated from sagittal radiography of the thoracic column using Cobb's method and by means of the flexicurve method. The intraclass correlation coefficient (ICC) between two measurements was 0.91 .

Estimates of intertrial and interrate reliability of spine curvature measures acquired using the digital inclinometer and flexicurve ruler also were similar [29].

As different instruments with different methods are used in each study and because of subject's variation in different regions and races, there is a wide range of results for mean thoracic kyphosis. In nine mentioned studies on 10 to 20 years of age boys, it ranged from $25.11^{\circ}$ to $53.3^{\circ}$. Our results in the present study (from $34.41^{\circ}$ to $36.49^{\circ}$ ) are in agreement with them.

Generally, the values driven by radiographic method are obviously more than those taken by noninvasive methods. This may be due to difference in measuring techniques.

\section{Conclusions}

Using flexicurve ruler in measuring thoracic kyphosis which is ideal for systematic population studies, a norm was established for male students aged 13 to 18 years old which can be used as normal values for local and regional purposes. Our results are in agreement with previous reports.

\section{Conflict of Interests}

The authors declare that there is no conflict of interests regarding the publication of this paper.

\section{Acknowledgment}

This study was supported by grants from Kermanshah University of Medical Sciences.

\section{References}

[1] S. Standring, Gray's Anatomy, Elsevier Churchill Livingstone, Edinburgh, UK, 39th edition, 2005.

[2] G. J. Fon, M. J. Pitt, and A. C. Thies Jr., "Thoracic kyphosis: range in normal subjects," The American Journal of Roentgenology, vol. 134, no. 5, pp. 979-983, 1980.

[3] Y. Nishiwaki, Y. Kikuchi, K. Araya et al., "Association of thoracic kyphosis with subjective poor health, functional activity and blood pressure in the community-dwelling elderly," Environmental Health and Preventive Medicine, vol. 12, no. 6, pp. 246250, 2007.

[4] A. M. Briggs, T. V. Wrigley, E. A. Tully, P. E. Adams, A. M. Greig, and K. L. Bennell, "Radiographic measures of thoracic kyphosis in osteoporosis: cobb and vertebral centroid angles," Skeletal Radiology, vol. 36, no. 8, pp. 761-767, 2007.

[5] S. D. Ryan and L. P. Fried, "The impact of kyphosis on daily functioning," Journal of the American Geriatrics Society, vol. 45, no. 12, pp. 1479-1486, 1997.

[6] P. M. Murray, S. L. Weinstein, and K. E. Spratt, "The natural history and long-term follow-up of Scheuermann kyphosis," The Journal of Bone and Joint Surgery A, vol. 75, no. 2, pp. 236248, 1993.

[7] M. Di Bari, M. Chiarlone, D. Matteuzzi et al., "Thoracic kyphosis and ventilatory dysfunction in unselected older persons: an epidemiological study in Dicomano, Italy," Journal of the American Geriatrics Society, vol. 52, no. 6, pp. 909-915, 2004.

[8] R. Cailliet, Shoulder Pain, F.A. Davis Company, Philadelphia, $\mathrm{Pa}$, USA, 1991.

[9] E. Ayub, "Posture and the upper quarter," in Physical Therapy of the Shoulder, R. Donatelli, Ed., pp. 81-90, Churchill Livingstone, Melbourne, Australia, 1991.

[10] D. H. Watson and P. H. Trott, "Cervical headache: an investigation of natural head posture and upper cervical flexor muscle performance," Cephalalgia, vol. 13, no. 4, pp. 272-284, 1993.

[11] O. Grimsby and J. Gray, "Interrelation of the spine to the shoulder girdle," in Physical Therapy of the Shoulder, R. Donatelli, Ed., pp. 95-129, Churchill Livingstone, New York, NY, USA, 1997.

[12] J. Gray and O. Grimsby, "Interrelationship of the spine, rib cage, and shoulder," in Physical Therapy of the Shoulder (Clinics in Physical Therapy), R. Donatelli, Ed., pp. 133-185, Churchill Livingston, Edinburgh, UK, 2004.

[13] S. Willner and B. Johnson, "Thoracic kyphosis and lumbar lordosis during the growth period in children," Acta Paediatrica Scandinavica, vol. 72, no. 6, pp. 873-878, 1983.

[14] S. L. Propst Proctor and E. E. Bleck, "Radiographic determination of lordosis and kyphosis in normal and scoliotic children," Journal of Pediatric Orthopaedics, vol. 3, no. 3, pp. 344-346, 1983.

[15] S. A. Voutsinas and G. D. MacEwen, "Sagittal profiles of the spine," Clinical Orthopaedics and Related Research, vol. 210, pp. 235-242, 1986. 
[16] M. Bernhardt and K. H. Bridwell, "Segmental analysis of the sagittal plane alignment of the normal thoracic and lumbar spines and thoracolumbar junction," Spine, vol. 14, no. 7, pp. 717721, 1989.

[17] P. Stagnara, J. C. De Mauroy, and G. Dran, "Reciprocal angulation of vertebral bodies in a sagittal plane: approach to references for the evaluation of kyphosis and lordosis," Spine, vol. 7, no. 4, pp. 335-342, 1982.

[18] E. H. Boseker, J. H. Moe, R. B. Winter, and S. E. Koop, "Determination of "normal" thoracic kyphosis: a roentgenographic study of 121 "normal" children," Journal of Pediatric Orthopaedics, vol. 20, no. 6, pp. 796-798, 2000.

[19] J. W. Youdas, J. H. Hollman, and D. A. Krause, “The effects of gender, age, and body mass index on standing lumbar curvature in persons without current low back pain," Physiotherapy Theory and Practice, vol. 22, no. 5, pp. 229-237, 2006.

[20] S. Hoppenfeld, Physical Examination of the Spine and Extremities, New Edition, Appleton-Century-Crofts, New York, NY, USA, 1976.

[21] J.-M. Mac-Thiong, É. Berthonnaud, J. R. Dimar II, R. R. Betz, and H. Labelle, "Sagittal alignment of the spine and pelvis during growth," Spine, vol. 29, no. 15, pp. 1642-1647, 2004.

[22] M. S. Poussa, M. M. Heliövaara, J. T. Seitsamo, M. H. Könönen, K. A. Hurmerinta, and M. J. Nissinen, "Development of spinal posture in a cohort of children from the age of 11 to 22 years," European Spine Journal, vol. 14, no. 8, pp. 738-742, 2005.

[23] A. Cil, M. Yazici, A. Uzumcugil et al., "The evolution of sagittal segmental alignment of the spine during childhood," Spine, vol. 30, no. 1, pp. 93-100, 2005.

[24] C. A. Giglio and J. B. Volpon, "Development and evaluation of thoracic kyphosis and lumbar lordosis during growth," Journal of Children's Orthopaedics, vol. 1, no. 3, pp. 187-193, 2007.

[25] Y. Takemitsu and Y. Harada, "Anteroposterior spinal curvature of normal growing children," Journal of the Japanese Orthopaedic Association, vol. 51, pp. 795-801, 1977.

[26] R. Vedantam, L. G. Lenke, J. A. Keeney, and K. H. Bridwell, "Comparison of standing sagittal spinal alignment in asymptomatic adolescents and adults," Spine, vol. 23, no. 2, pp. 211-215, 1998.

[27] T. Widhe, "Spine: posture, mobility and pain. A longitudinal study from childhood to adolescence," European Spine Journal, vol. 10, no. 2, pp. 118-123, 2001.

[28] F. Teixeira and G. Carvalho, "Confiabilidade e validade das medidas da cifose toraica atraves do metodo flexicurva," Revista Brasileira de Fisioterapia, vol. 11, pp. 199-204, 2007.

[29] N. J. Macintyre, L. Bennett, A. M. Bonnyman, and P. W. Stratford, "Optimizing reliability of digital inclinometer and flexicurve ruler measures of spine curvatures in postmenopausal women with osteoporosis of the spine: an illustration of the use of generalizability theory," ISRN Rheumatology, vol. 2011, Article ID 571698, 8 pages, 2011. 


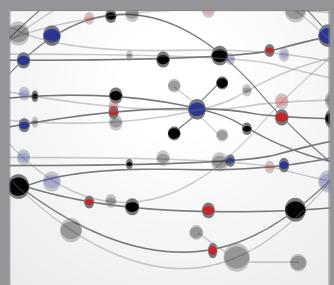

The Scientific World Journal
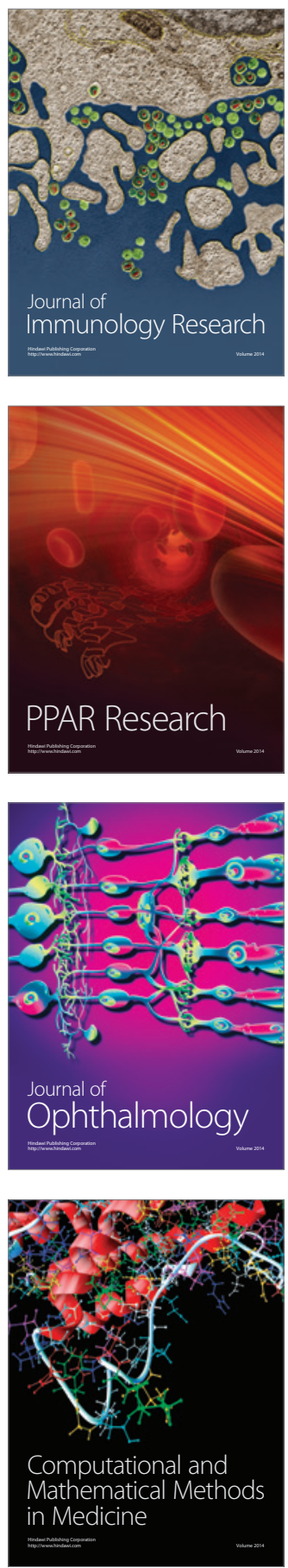

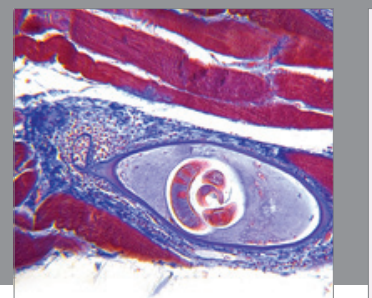

Gastroenterology

Research and Practice
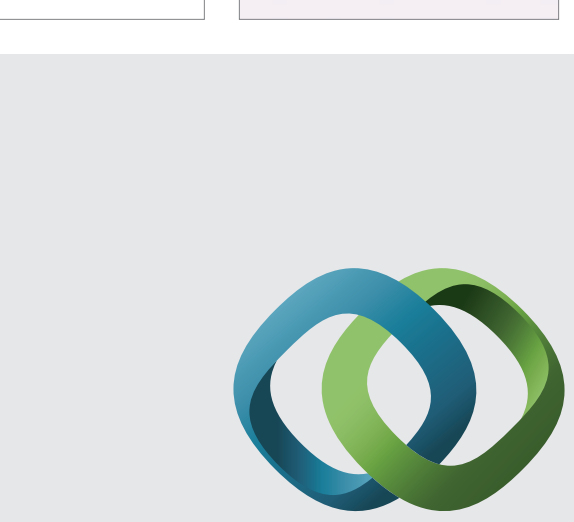

\section{Hindawi}

Submit your manuscripts at

http://www.hindawi.com
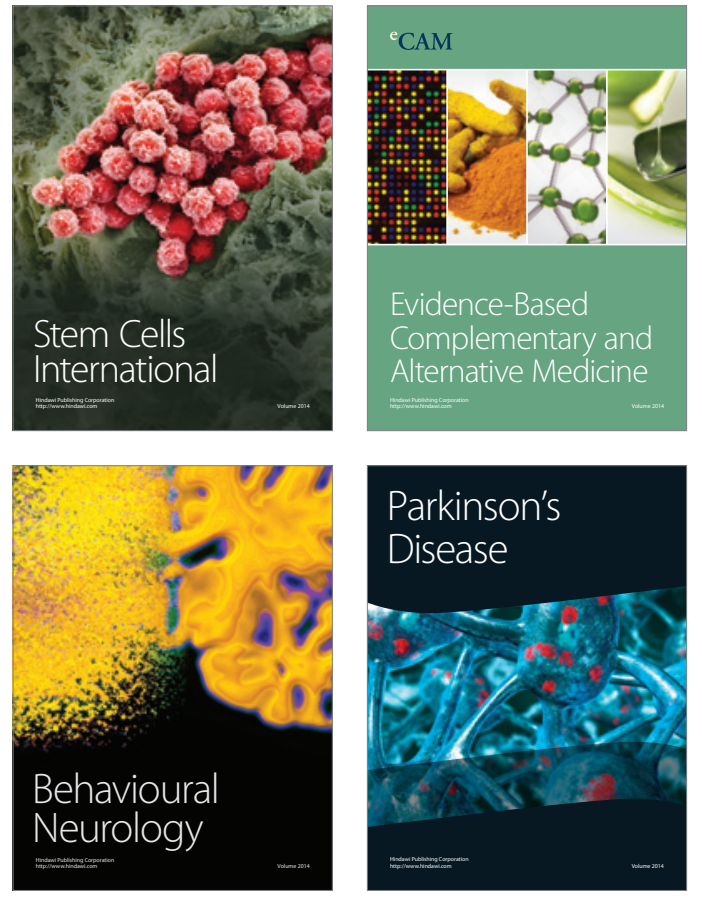
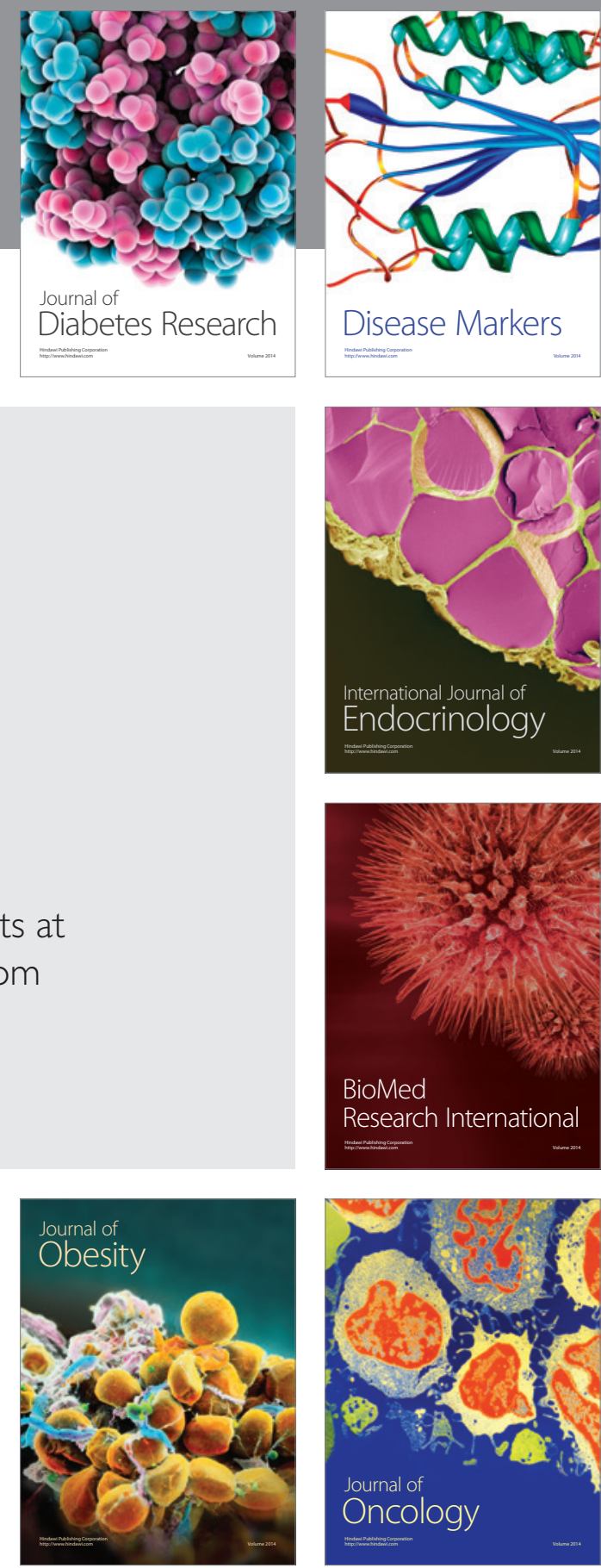

Disease Markers
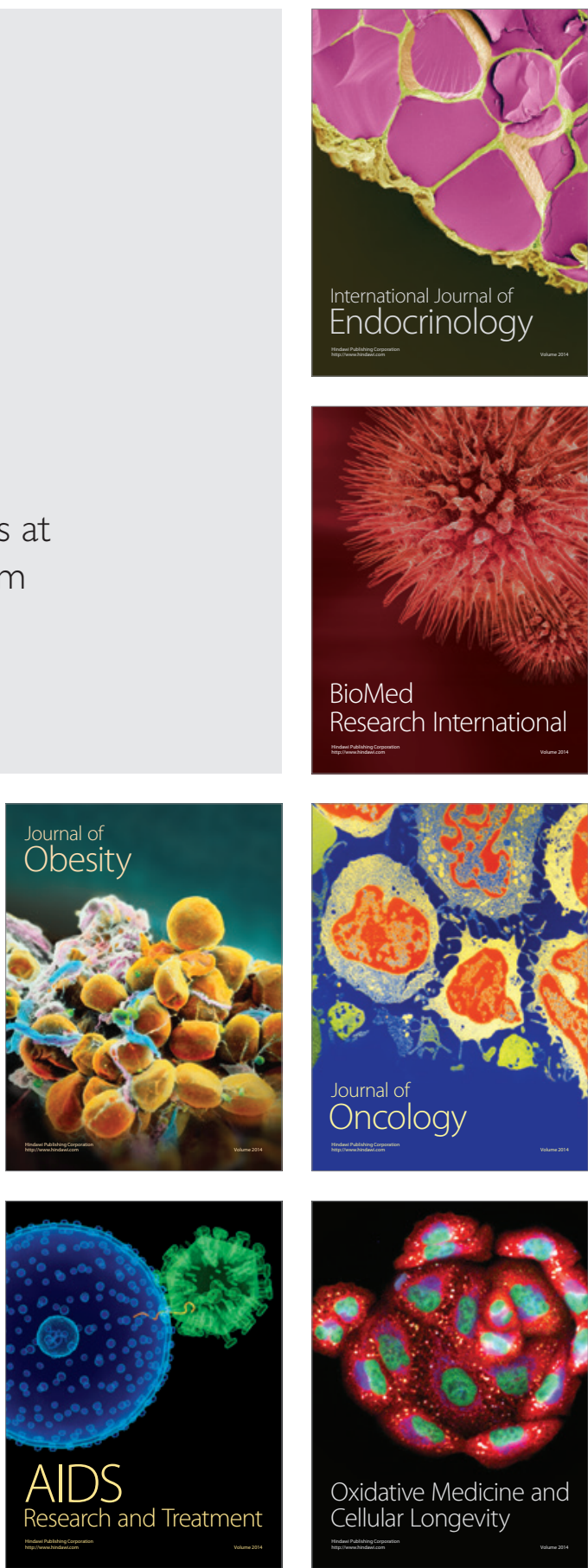